\title{
A nucleotide-dependent conformational switch \\ controls human IMP dehydrogenase polymerization \\ to modulate their catalytic activity
}

David Fernández-Justel ${ }^{1}$, Rafael Núñez ${ }^{2}$, Jaime Martín-Benito ${ }^{3}$, David Jimeno ${ }^{4}$, Adrián González-López ${ }^{1}$, Eva María Soriano ${ }^{1,5}$, José Luis Revuelta ${ }^{1 *}$ \& Rubén M Buey $^{1^{*}}$

${ }^{1}$ Metabolic Engineering Group, Dpto. Microbiología y Genética. Universidad de Salamanca. Campus Miguel de Unamuno, 37007, Salamanca, Spain

${ }^{2}$ Centro de Investigaciones Biológicas (CIB), Spanish National Research Council (CSIC), Ramiro de Maeztu 9, 28040 Madrid, Spain

${ }^{3}$ Centro Nacional de Biotecnología (CNB), Spanish National Research Council (CSIC), Darwin 3, 28039 Madrid, Spain

${ }^{4}$ Instituto de Biología Molecular y Celular del Cáncer (CSIC-Universidad de Salamanca). Campus Miguel de Unamuno, 37007, Salamanca. Spain

${ }^{5}$ Present address: Institute for Cardiovascular and Metabolic Research, School of Biological Sciences, Harborne building, University of Reading, Whiteknights, Reading, RG6 6AS, United Kingdom

"Correspondence to J.L.R. (revuelta@usal.es) or R.M.B.

E-mail: ruben.martinez@usal.es

Phone: +34 666506019

Address: Lab 233. Edificio Departamental. Campus Miguel Universidad de Salamanca.de Unamuno, 37007, Salamanca, Spain 


\section{Abstract}

IMP dehydrogenase (IMPDH) catalyzes the rate-limiting step in the de novo GTP biosynthetic pathway and plays essential roles in cell proliferation. As a clinical target, IMPDH has been studied for decades but it has only been within the last years that we are starting to understand the complexity of the mechanisms of its physiological regulation. Here, we report structural and functional insights into how adenine and guanine nucleotides control a conformational switch that modulates the assembly of the two human IMPDHs into cytoophidia and allosterically regulates their catalytic activity. In vitro reconstituted micron-length cytoophidia show catalytic activity comparable to unassembled IMPDH but, in turn, are more resistant to GTP/GDP allosteric inhibition. Therefore, IMPDH cytoophidia formation facilitates the accumulation of high levels of guanine nucleotides when the cell requires it. Finally, we demonstrate that most of the IMPDH retinopathy-associated mutations abrogate GTP/GDP-induced allosteric inhibition and alter cytoophidia dynamics.

\section{$\underline{\text { Keywords }}$}

IMP dehydrogenase, cytoophidia, conformational switch, allosteric regulation, X-ray crystallography and scattering 


\section{Introduction}

Inosine 5'-monophosphate dehydrogenase (IMPDH) is the enzyme that catalyzes the rate-limiting step in the de novo guanine nucleotide biosynthetic pathway, which plays a crucial role in the regulation of the cellular pools of purine nucleotides. Therefore, IMPDH is a key enzyme involved in the control of cell viability, division and proliferation. Indeed, IMPDH is the target of a diverse number of drugs presently used in clinical chemotherapy such as immunosupressors, antivirals, antitumorals or antibiotics [1].

IMPDH is composed of an archetypal TIM barrel catalytic domain and a Bateman regulatory domain, which is inserted within a loop of the catalytic module. In solution, the basic units of most IMPDHs are homotetramers that can dimerize in different ways to form octamers or higher order oligomers [2-7].

Despite the fact that IMPDH is a therapeutic target with important clinical relevance that has been widely studied during the last decades [1], the available information on its physiological regulation is rather limited. More specifically, the molecular details on how the regulatory and catalytic domains communicate to modulate the catalytic activity remain mostly unclear. Indeed, it has been only since the last few years that we are beginning to understand the mechanistic and structural details of IMPDH allosteric regulation [2, 3, 5-7].

Using the IMPDH enzyme from the industrial fungus Ashbya gossypii as a model, we have recently reported a molecular mechanism by which purine nucleotides allosterically modulate the catalytic activity. The competition of adenine or guanine nucleotides for their binding sites within the regulatory Bateman domain controls a conformational switch that modulates the catalytic activity by shifting from extended to compacted IMPDH octamers. Extended, ATP-induced octamers are fully active, while the catalytic activity of GTP/GDPinduced compacted octamers is significantly reduced [6, 7]. The relevance of this allosteric mechanism of regulation is stressed by the observation that most of the described missense mutations in human IMPDH associated to severe retinopathies, such as Retinitis Pigmentosa and Leber Congenital Amaurosis [8], map into the nucleotide binding sites of the Bateman regulatory domain. Based on these results, it was proposed that the retinopathy-associated 
mutations might interfere with nucleotide binding and disrupt the allosteric which is in agreement with the dominant hereditary character of these mutations [7].

Adding more complexity to the mechanisms of IMPDH regulation, the human enzyme has been reported to polymerize into mesoscale intracellular assemblies such as purinosomes [9] and micrometric structures denoted as rod and rings or cytoophidia [4, 10, 11]. Thereby, human IMPDH, as well as CTP synthase (CTPS) and some other metabolic enzymes, possess the particular ability to self-assemble into higher order structures. This macromolecular organization has been suggested to modulate enzyme activity and play important roles in regulating cell metabolism by controlling intracellular nucleotide homeostasis [12, 13]. Human IMPDH, in response to intracellular guanine-nucleotide depletion, assembles into micron-length cytoophidia that disappear once the guanine-nucleotide levels are restored. Remarkably, the physiological functions of IMPDH cytoophidia remain unclear, although it has been reported that polymerization correlates with rapid cell proliferation [11, 14, 15]. Nonetheless, the in vitro reconstituted polymers show identical activity for polymerized and non-assembled human IMPDH [5]. To reconcile these results, it has been recently speculated that filamentous IMPDH might be more resistant to GTP/GDP inhibition, facilitating the intracellular accumulation of GTP when required [15], but no experimental evidence supporting this hypothesis has been obtained. Moreover, there is an evident lack of information regarding the structural details and the molecular mechanisms governing the dynamics of IMPDH cytoophidia assembly in vitro. Indeed, to our best knowledge, the in vitro reconstituted IMPDH filaments studied so far consist of a few particles organized into chains polymerized by a back-to-back assembly mechanism [2, $4,5]$ that do not resemble the micrometer-long fibrillar IMPDH cytoophidia that are observed in cells [10,11, 16-21]

In this study, we report the in vitro reconstitution of human IMDPH cytoophidia and their structural and functional characterization. We show that micron-length human IMPDH cytoophidia spontaneously assemble in vitro under macromolecular crowding conditions in the absence of nucleotides but 
disassemble when either GTP or ATP are present in the solution, resembling what is observed within cells. The in vitro reconstituted IMPDH cytoophidia are catalytically functional, with similar activity to non-assembled IMPDH, but they are significantly more resistant to GTP/GDP allosteric inhibition. Our results show that the cytoophidia dynamics and the allosteric regulation of the catalytic activity are tightly linked to the purine nucleotide-controlled conformational switch that we have previously reported for the Ashbya gossypii fungal IMPDH $[6,7]$. They further provide a plausible structural mechanism for the nucleotidedependent dynamics of cytoophidia assembly. Finally, we present the highresolution crystal structures of HsIMPDH2 complexed to GTP and GDP and demonstrate that most of the retinopathy-associated missense mutations map into the allosteric nucleotide binding sites of human IMPDH, altering nucleotide binding and subsequently abrogating GTP/GDP-induced allosteric inhibition. Additionally, these mutations also alter cytoophidia dynamics, suggesting a potential pathological role for these assemblies that deserves to be further investigated.

Altogether, the results presented here yield valuable information to understand the complex mechanisms of physiological regulation of IMPDH in humans, as well as the roles that these mechanisms might have in the development of certain retinopathies.

\section{Results}

A nucleotide-controlled conformational switch operates in both human IMPDH isoforms. By using the IMPDH enzyme from the industrial fungus Ashbya gossypii, we have recently reported a conformational switch that modulates the catalytic activity in response to the binding of purine nucleotides within the Bateman regulatory domain: adenine nucleotides (ATP, ADP and AMP) induce active IMPDH octamers in an extended (-115 $\AA$-long) conformation, while guanine nucleotides (GTP and GDP) induce inhibited octamers in a compact (95 A-long) conformation [6]. Experimental evidence of the existence of this conformational switch in the isoform 2 of human IMPDH (HsIMPDH2) was recently reported by using electron microscopy on ATP and GTP-induced 
helical protofilaments [5]. Here, we have further investigated the nucleotideconformational switch in both human IMPDH isoforms (HsIMPDH1 and HsIMPDH2) in vitro by using Small Angle X-ray solution Scattering (SAXS).

HsIMPDH1 and HsIMPDH2 wild type proteins at the concentration used for the SAXS experiments $(2.5 \mathrm{mg} / \mathrm{mL})$ are mostly octamers in solution but the addition of nucleotides induces stochastic oligomerization (see below) that results in poly-disperse solutions [2, 4, 5], which are not appropriate for SAXS analysis. Thereby, we made use of the missense mutation $\mathrm{Y} 12 \mathrm{~A}$ that has been previously reported to abrogate polymerization of HsIMPDH2 in vitro and in cultured cells [5]. Indeed, in the absence of nucleotides, HsIMPDH1-Y12A and HsIMPDH2-Y12A mutant enzymes remained as monodisperse tetramers in solution, as demonstrated by size-exclusion chromatography (Supplemental Figure $1 \mathrm{~A}$ ). This observation indicates that the octamers that exist in solution in the absence of nucleotides are most probably formed by the back-to-back interaction of the catalytic domains of two tetramers with a crucial role for residue $\mathrm{Y} 12$, as suggested in previous reports $[2,5]$.

The addition of ATP or GDP to HsIMPDH1/2-Y12A induces the association of two tetramers to form octamers (Figures $1 A$ and $1 B$ ). Remarkably, the SAXS profiles of both human IMPDH isoforms resemble those described for the A. gossypii ATP or GDP-induced octamers (Supplemental Figures 1B-D), demonstrating that the conformational switch previously proposed for the fungal Ashbya gossypii AgIMPDH [6] is also present in both isoforms of the human IMPDH enzyme.

As shown in Supplemental Figures 1E-F, ATP induces the formation of octamers more potently than GDP for both HsIMPDH1 and HsIMPDH2. Interestingly, significant differences are observed between both isoforms. In HsIMPDH1, the GDP-induced inhibition of the catalytic activity correlates with octamer compaction (Figure 1C). In contrast, GDP-induced compact octamers of HsIMPDH2 are formed before inhibition occurs, both in the absence or presence of ATP in the solution (Figure 1D), highlighting mechanistic differences between both human isoforms that might be relevant in vivo. 
In summary, our data show that the nucleotide-controlled conformational human IMPDH isoforms, where the binding of ATP or GDP induces octamers, formed by the interaction of the Bateman domains of two tetramers. The presence of adenine nucleotides in the solution induce octamers with an extended conformation that are catalytically active, while guanine nucleotides induce the compaction of these octamers, significantly inhibiting their enzymatic activity.

Retinopathy-associated mutations abrogate the allosteric inhibition of human $I M P D H$. To gain insights into the binding modes of purine nucleotides to the Bateman domain of human IMPDH, we attempted to obtain high resolution structural information by X-ray crystallography. This issue is especially relevant since, based on the A. gossypii fungal IMPDH structure, we have previously proposed that most of the retinopathy-associated missense mutations in HsIMPDH1 alter GTP/GDP-mediated allosteric inhibition [7]. Thereby, we solved the high-resolution crystal structures of HsIMPDH2 in complex with GTP and GDP, which are essentially identical (Supplemental Figure 2A). A detailed description of these structures can be found in the Supplemental Information. Both structures unequivocally demonstrate that most of the retinopathyassociated mutations either: i) placed at the interface of two interacting Bateman domains: R224P, L227P and R231P (Supplemental Figure 2C) or ii) are directly involved in nucleotide binding at the allosteric sites of the Bateman domain: D226N for GTP/GDP2 (Supplemental Figure 3B) and N198K and K238E for GTP3/GDP3 (Supplemental Figure 3C).

We then introduced most of the missense mutations described in the literature [8] into HsIMPDH1 and purified the mutant enzymes for their analyses in vitro. Our results showed that the mutants had catalytic activity in vitro that fell within the same range (between 1.1 and 0.6 -fold the activity of the WT; Figure 2) as the WT enzyme. ATP showed no significant effect on the catalytic activity of both the WT and the mutant enzymes (between 1.0 and 0.6 -fold the activity of the enzyme without nucleotide; Figure 2). On the other hand, those mutations that map within the Bateman domain nucleotide binding sites (N198K, D226N and K238E; Supplemental Figure 3) or at the interphase 
between the Bateman domains (R224P, L227P and R231P; Supplemental Figure $2 \mathrm{C}$ ) cannot be allosterically inhibited in vitro neither by GTP/GDP (Figure 2 ), which thereby results in constitutively activated mutant enzymes.

In vitro reconstitution of micron-length human IMPDH cytoophidia assembly. We then investigated in vitro the ability of IMPDH to reversibly assemble into mesoscale structures that have been described to form within cells in response to the depletion of the intracellular guanine nucleotide pool $[10,19,21]$. It is important to stress here that several reports have shown that ATP and GTP induce the formation of IMPDH polymers in vitro [3-5], but these polymers consist of a few IMPDH octamers stacked back-to-back and organized into single filaments. Thereby, these polymers do not represent the IMPDH micronlength filamentous bundles that have been reported to form in cells $[10,18,19$, 21].

These data suggest that an important factor is missing in vitro, but what could be this factor? An initial hint came from the published purification protocol of recombinant human IMPDH enzymes from E. coli [21]. This protocol uses 1.5 M urea to keep the protein "soluble" in the supernatant after the initial centrifugation of the bacterial cell extracts. However, 1.5 M urea concentration is much lower than the midpoint of the urea-induced denaturation curve (approximately 5M [22]), which indicates that there is no need to denature the IMPDH to "solubilize" it. We then hypothesized that IMPDH properly folds in the cytoplasm of $E$. coli, however the high background concentration of macromolecules in the initial cell extracts favored the formation of large IMPDH assemblies that readily pelleted during a soft centrifugation.

We therefore tested the influence of macromolecular crowding on IMPDH filament assembly in vitro under different experimental conditions by monitoring protein assembly with turbidimetry. Our results showed that at high Ficoll-70 concentrations both human IMPDH isoforms (HsIMPDH1 and HsIMPDH2) spontaneously assemble into large polymers, as indicated by the large increase in the turbidity of the solution (Figure $3 A$ and Supplemental Figure $4 A$ ), and further corroborated by electron microscopy (EM; upper panels of Figure $3 B$ and Supplemental Figure 4B). At Ficoll-70 concentrations $\geq 150 \mathrm{mg} / \mathrm{mL}$, most of the IMPDH is included within these polymers. These results led us to conclude 
that the formation of micron-length cytoophidia is due to the macromolecular crowding conditions, since mesoscale polymerization can be detected neither at low crowding agent concentrations nor in its absence.

As a negative control, we used the Y12A missense mutation that has been previously reported to abrogate polymerization of HsIMPDH2 in cultured cells [5]. In accordance to this report, we were not able to detect significant assembly of the HsIMPDH2-Y12A mutant in vitro in dilute solutions or in crowding conditions (Figure $3 \mathrm{C}$ ). In contrast, we observed significant polymerization of the HsIMPDH1-Y12A mutant under macromolecular crowding conditions, although to a lower extent than the wild-type protein (Figure 3C). These results agree with previous reports that describe a higher tendency to polymerize of HsIMPDH1 than HsIMPDH2 [23]. Additionally, we tested whether the IMPDH enzymes from different organisms, other than humans, can also form cytoophidia in vitro. Within Figure $3 \mathrm{C}$, it can be observed in that, in contrast to the two human isoforms, the eukaryotic Ashbya gossypii (AgIMPDH), the bacterial E. coli (EcIMPDH) and B. subtilis (BsIMPDH) enzymes are unable to form cytoophidia in our experimental conditions.

To further corroborate that eukaryotic AgIMPDH does not form cytoophidia, we ectopically overexpressed this gene in HeLa and HEK293T cells. As it is shown in Supplemental Figure 4C, in contrast to HsIMPDH2 that showed a large amount of cytoophidia in essentially all the transfected cells, no detectable polymer formation was observed for AgIMPDH, despite the large amount of protein observed into the cytoplasm. Thereby, our results demonstrate that the ability of human IMPDH to form cytoophidia is not universal, since not all -eukaryotic or prokaryotic- IMPDH enzymes are able to assemble into large aggregates. In agreement with our results, other authors could neither find IMPDH polymers in the model yeast S. cerevisiae [24].

The huge polymers of human IMPDH that formed in vitro under macromolecular crowding conditions are composed of octamers that associate into bundles of twisted and curved filaments that intricate into three dimensions without apparent regular or defined structure (red rectangle in the upper right panel of Figure 3B and upper right panel of Supplementary Figure 4B). Nonetheless, ordered bundles of parallel protofilaments were eventually 
observed within the huge polymers in some of the HsIMPDH2 micrographs (blue rectangle in the upper right panel in Figure 3B). Direct distance measurements and power spectra analysis by fast-fourier transform (FFT) of the protofilaments within these bundles showed a repetitive longitudinal spacing of $58.2 \pm 0.4 \AA$. This longitudinal spacing is compatible with the expanded ATPinduced $\sim 115 \AA$-long octamers previously observed by X-ray crystallography for AgIMPDH [6] and by SAXS for human IMPDH.

Remarkably, when ATP or GDP were present, the turbidity of the solution was significantly lower than in their absence (Figure $3 \mathrm{~A}$ and Supplemental Figure 4A), indicating that these nucleotides inhibited IMPDH cytoophidia assembly. The EM analysis of the polymers formed in the presence of purine nucleotides revealed either isolated octamers or helical oligomers, extended for ATP and compacted for GDP (Figure 3B and Supplementary Figure 4B). A detailed analysis of the polymers formed in the presence of purine nucleotides can be found in the Supplemental Information, including the 3D reconstructions of ATP and GDP-induced protofilaments formed under macromolecular crowding conditions (Supplementary Figure 6).

Moreover, once the cytoophidia were spontaneously formed in vitro, the addition of either ATP or GDP depolymerized them, but the extent of this depolymerization strongly depended on the incubation time before nucleotide addition, i.e. the cytoophidia maturation time [18]. The more mature the cytoophidia are, the longer it takes to depolymerize them by GDP or ATP (Supplemental Figure 5).

In summary, both human IMPDH isoforms spontaneously assemble in vitro into micron-length cytoophidia under macromolecular crowding conditions that resemble those found in the cytoplasm. Under these conditions, the presence of purine nucleotides in the solution strongly reduce IMPDH cytoophidia formation, by both inhibiting assembly and inducing disassembly. Thus, our experimental setup reconstitutes in vitro the reversible nucleotidecontrolled cytoophidia assembly phenomenon observed in cells.

Retinopathy-associated missense mutations in IMPDH1 alter the nucleotidedependent cytoophidia dynamics in vitro. We next studied the nucleotide- 
dependent dynamics of cytoophidia assembly in vitro for the IMPDH1 enzymes containing the retinopathy-associated mutations. All the mutants have the ability to spontaneously assembly into cytoophidia to a similar extent than the wild type enzyme (Figure 4). Nonetheless, those mutants where the GTP/GDP allosteric inhibition is abrogated have also altered nucleotide-controlled regulation of cytoophidia assembly (Figure 4) and disassembly (Supplemental Figure 7A). The only exception is the mutant N198K that cannot be allosterically inhibited by GTP/GDP but possesses cytoophidia dynamics comparable to the wild-type enzyme (Figure 4 and Supplemental Figure 7A). These data might indicate that the non-canonical guanine nucleotide binding site is needed for allosteric inhibition mediated by GTP/GDP, but is not implied in the regulation of cytoophidia assembly by nucleotides.

Remarkably, our data on cytoophidia assembled in vitro perfectly agree with a recent report that showed that HsIMPDH1 mutant enzymes R224P, D226N and R231P formed irreversible cytoophidia in transfected HEK293T cells when treated with guanosine [14].

IMPDH cytoophidia are catalytically active in vitro but more resistant to GTP/GDP inhibition. We next studied the effects of the polymerization into micron-length cytoophidia on the catalytic activity in vitro. To perform these experiments, we used as control the $\mathrm{Y} 12 \mathrm{~A}$ mutants that have compromised polymerization ability (Figure $3 \mathrm{C}$ ). Remarkably, we observed no significant differences between the catalytic activities of the Y12A mutants and the wildtype enzymes, neither in crowded nor in non-crowded solutions (Supplemental Table 2, Figures $5 \mathrm{~A}$ and $5 \mathrm{~B}$ ). The slight increase in the $\mathrm{K}_{M}$ values between crowded and non-crowded conditions are most probably due to excluded volume and substrate limited diffusion effects, produced by the high concentrations of Ficoll-70. Indeed, similar differences in the $K_{M}$ and $K_{1 / 2}$ values have been reported for a number of enzymes using similar crowding agents [25].

As an additional control, just before the measurements, we centrifuged the samples $(5.000 \times \mathrm{g}$ for $10 \mathrm{~min}$ ), so that the cytoophidia were pelleted and the tetramers, octamers and small oligomers remained in the supernantant. The catalytic activity in the supernatant of the wild-type enzymes under crowding 
conditions was significantly reduced (Supplemental Figure 7B), demonstrating that most of the catalytic activity comes from the IMPDH cytoophidia. Additionally, most of the $\mathrm{Y} 12 \mathrm{~A}$ mutant enzyme, that have compromised cytoophidia formation (Supplemental Figure $7 \mathrm{C}$ ), cannot be pelleted using these centrifugation conditions, therefore leaving the activity in the supernatant comparable to the activity prior to centrifugation (Supplemental Figure 7B). From these experiments, we can conclude that IMPDH polymerization into micron-length cytoophidia did not significantly affect the catalytic activity in our in vitro experimental conditions.

Since GTP and GDP promote both cytoophidia disassembly and catalytic activity inhibition, it can be hypothesized that IMPDH polymerization might serve to fine-tune the allosteric regulation of the enzyme. To test this hypothesis, we studied how GDP affects the catalytic activity of the cytoophidia assembled in our in vitro system. As observed in Figure 5C, non-mature cytoophidia (without incubation before GDP addition) demonstrated and inhibition of the catalytic activity while being depolymerized (red data) that showed no significant differences to the inhibition of the unassembled protein (green data). In contrast, the cytoophidia that had been pre-incubated for 2 hours before GDP addition, were more resistant to GDP-induced depolymerization (see also Supplemental Figure 5) and to a certain extent were resistant to GDP allosteric inhibition (blue data). As expected, this behavior can also be observed for HsIMPDH2 but to a lesser extent (Figure 5D), given that HsIMPDH2 is more sensitive to GDP-induced depolymerization than HsIMPDH1 (Supplemental Figure 5). Moreover, HsIMPDH2 was depolymerized at lower GDP concentrations than those required to inhibit its catalytic activity (Figure 5D). This observation agrees with the data presented above showing that GDP induces octamer compaction at lower concentrations than those required for catalytic activity inhibition (Figure 1D).

\section{Discussion}

Despite there is wide knowledge on the enzymatic reaction mechanisms of IMPDH and its inhibition by a variety of molecules, there is an evident lack of 
information on its physiological mechanisms of regulation. Indeed, it has only evidences on how the signal triggered by the binding of nucleotides to the regulatory Bateman domain is transmitted to the catalytic domain to modulate the enzymatic activity of IMPDH. To this respect, it has been recently described a conformational switch -controlled by purine nucleotides- that modulates the catalytic activity of eukaryotic IMPDH enzymes. The switch was first proposed by crystallography and X-ray scattering using the enzyme AgIMPDH, from the industrial fungus Ashbya gossypii [6] and was recently observed by electron microscopy with HsIMPDH2, the second isoform of human IMPDH [5]. We further demonstrate here that this conformational switch operates in both human IMPDH isoforms, suggesting that it might be conserved within all eukaryotic IMPDH enzymes. Moreover, a similar conformational switch, modulated by adenine nucleotides, has also been reported for some prokaryotic IMDPHs [2], despite the fact that these enzymes are not regulated by guanine nucleotides [7]. This observation suggests that the conformational switch is inherent to the acquisition of the Bateman regulatory domain, before the divergence of prokaryotic and eukaryotic enzymes. Nevertheless, the latter evolved separately to generate an additional non-canonical nucleotide binding site that allows the inhibition of the enzyme by GTP and GDP, the end products of the IMPDH's metabolic pathway.

Remarkably, we have found functional differences between the two human isoforms of IMPDH, where GTP/GDP-mediated allosteric inhibition of the catalytic activity is nicely correlated with octamer compaction in HsIMPDH1. In contrast, HsIMPDH2 octamer compaction occurs at lower GTP/GDP concentrations than those required for catalytic inhibition. These data, observed both in HsIMPDH2- Y12A (Figure 1D) and HsIMPDH-WT (Figure 5D), indicate that additional features, other than octamer compaction itself, are required to inhibit HsIMPDH2. Possibly, the catalytic inhibition of IMPDH requires the binding of GTP/GDP to the three allosteric sites within the Bateman domain, while octamer compaction can happen with only a partial occupancy of these sites. Thereby, distinct affinities of GTP/GDP among the three nucleotidebinding sites of HsIMPDH1 and HsIMPDH2 might be the cause of the observed 
differences. Additionally, our data show that HsIMPDH1 has a significantly higher tendency to filament than HsIMPDH2, in accordance to previous reports [23]. Future experimental studies will elucidate whether the different sensitivities to GTP/GDP-mediated allosteric inhibition and/or the different propensities to polymerize of the human IMPDH isoforms might have physiological consequences.

Presently, it is clearly accepted that the filamentation of enzymes to form cytoophidia serves as a general mechanism for the regulation of metabolism by fine-tuning protein functional and structural properties $[12,13]$. Indeed, enzyme filamentation seems to be rather extended with more than 20 metabolic enzymes reported to filament in $S$. cerevisae [24]. Perhaps the most representative examples of cytoophidia-forming enzymes are those that catalyze the limiting steps in the de novo biosynthetic pathways of pyrimidine and purine nucleotides, CTP synthase (CTPS) and IMPDH, respectively.

Human CTPS filaments lock the enzyme into an active conformation, providing a mechanism for increasing the catalytic activity in response to metabolic state [26]. Similarly, human IMPDH cytoophidia formation correlates with rapid cell proliferation $[11,14,15]$, however the in vitro reconstituted polymers show identical activity for both polymerized and non-assembled IMPDH [5]. In any case, the reported structural and functional data for IMPDH cytoophidia in vitro are based on polymers, induced by ATP or GTP, that are formed by the repeated back-to-back stacking of tetramers and octamers into single filaments [4, 5], which do not represent the micrometer long and thick bundles observed in cells $[10,19]$. The results presented here demonstrate that these polymers do not represent cytoophidia but rather the remnants of their depolymerization. Our experimental conditions yield IMPDH polymer bundles in vitro with similar dimensions to the micron-length cytoophidia found in cells. Moreover, the dynamics of these polymers is modulated by purine nucleotides in vitro in the same way as it has been observed in cells, as it will be discussed below. Thereby, our conditions allow a much better biochemical and structural characterization of this biological process.

The nucleotide-mediated steric self-compatibility of the Bateman interface and the highly symmetric structure of the octamers promote the 
assembly of IMPDH into the relatively thin and extended filaments that have been previously reported to form in vitro [4, 5]. Nonetheless, once these filaments are formed, they are not able to significantly associate side by side to form the bundles that constitute cellular cytoophidia. The higher order assembly into bundles of filaments proceeds only in the absence of nucleotides and seems to be a cooperative process, much less favorable than the nucleotideinduced single filament assembly is. Consistent with these observations, IMPDH assembly into micron-length cytoophidia depends on macromolecular crowding conditions, indicating that this assembly reaction is not only driven by enthalpy due to specific interface interactions, but rather is also driven by entropy as a consequence of excluded volume effects [27]. Indeed, in a crowded milieu, oligomerization is greatly favored to minimize the overall crowding by enhancing protein association, which can thereby reduce the excluded volume [28]. The cytoophidia assembled under these conditions are constituted by IMPDH octamers associated without an apparent order, although highly ordered specimens are also observed in the HsIMPDH2 micrographs. This observation indicates that the association of IMPDH octamers under crowding conditions confers high flexibility and dynamicity to the cytoophidia, which is in further agreement with the observed variety of shapes that easily inter-convert in cells.

Our in vitro results reconstitute what it is observed in cells: IMPDH assemble into micron-length cytoophidia upon guanine nucleotide depletion but cytoophidia disassemble after guanine levels recover. This observation might also apply for the adenine nucleotides, because it is assumed that the levels of the adenine and guanine nucleotide pools rise or decrease in parallel, at least to a certain extent. Several mechanisms explain the parallel changes in adenine and guanine nucleotide levels. Firstly, lowering the GTP levels might provoke a decrease in the adenine nucleotide levels through a dual action by: i) a reduced synthesis of AMP through adenylosuccinate synthetase, for which GTP serves as cofactor, and ii) a greater degradation of AMP by AMP deaminase, which is inhibited by GTP. Secondly, an interplay exists between the syntheses of AMP/ATP and GMP/GTP from IMP, where GTP is required to synthetize AMP 
at the level of adenylosuccinate synthase, and ATP is required for the synthesis of GMP at the level of GMP synthase.

How do nucleotides modulate cytoophidia formation? A plausible explanation is that adenine and guanine nucleotides induce IMPDH octamer conformations that allow a few octamers to stack back-to-back and form short lineal filaments $[4,5]$, but these filaments do not significantly associate laterally to form the large bundles that constitute the cytoophidia. In contrast, in the absence of purine nucleotides, human IMPDH adopts a conformation that under crowding conditions favors both filament formation and filament bundling. A schematic representation of this hypothesis is shown in Figure 6. In support of this hypothesis, three different conformations for eukaryotic IMPDHs have been reported (Figure 6): i) ATP-induced extended active octamers ( $\underline{\mathbf{5 M C P}},[6])$, ii) GTP/GDP-induced compacted inhibited octamers $\underline{6100}, \underline{610 M}$ in this work and $\underline{4 Z 87}$ [7]), and iii) extended octamers with no nucleotide bound at the Bateman domain ( $\underline{\mathbf{1 N F 7}}$ and $\underline{\mathbf{1 N F B}})$. The structures of the apo-enzyme and the ATPinduced IMPDH octamers have similar dimensions, but they significantly differ in the bending of the Bateman module that increases upon ATP binding (Figure 6). Therefore, it is then tempting to speculate that the less bent Bateman module of the apo-enzyme octamers favors the lateral packing of the single filaments that result in large micrometric bundles. On the contrary, the increased bending of the Bateman module, induced by the binding of either ATP or GTP/GDP to the allosteric sites, avoids the lateral association of octamers and single protofilaments that consequently avoids cytoophidia formation (Figure 6). Indeed, the crystal packing of 1NF7 (Supplemental Figure $8 \mathrm{~A})$ would be compatible with the repetition pattern observed in the ordered bundles of HsIMPDH2 found in some of the micrographs of the in vitro reconstituted cytoophidia (Figure 3B) and in a previous report [19], suggesting that this crystal might be mimicking the packing of IMPDH within the cytoophidia.

Our results demonstrate that assembly into cytoophidia does not significantly change the catalytic activity of purified IMPDH in vitro. These results agree with a previous report that could neither find differences in catalytic activity between a mutant unable to polymerize (HsIMPDH2-Y12A) and 
the wild type enzyme, both in vitro and in cultured cells [5]. In turn, IMPDH Remarkably, the more mature the cytoophidia (longer incubation times) are, the more resistant to GTP/GDP-induced octamer compaction they become and thereby more resistant to depolymerization and subsequent inhibition of the catalytic activity. In other words, the more mature a cytoophidia is, the longer it takes to depolymerize by GTP/GDP. At this point, it remains unknown how maturation time correlates to resistance to GDP-induced octamer compaction in vitro, but it is clear that it might have important physiological implications. The longer a cell remains in a state of guanine nucleotide deprivation, the longer it will take to recover, i.e. the longer IMPDH should work actively in the absence of negative feedback inhibition. Therefore, IMPDH cytoophidia formation and maturation might constitute a mechanism to temporal and spatially regulate the ability of IMPDH polymers to maintain especially high intracellular levels of guanine nucleotides when required, e.g. for active cell proliferation or after a pharmacological depletion of the guanine nucleotide pools. At this point, it is important to stress that this study was performed in vitro, from purified components, but it is envisaged that in vivo post-translational modifications and interaction partners will play an important role in the regulation of the dynamics of IMPDH cytoophidia assembly. Moreover, experiments with CTPS are presently being performed in our laboratory, since a coordinated response to simultaneously increase purine and pyrimidine nucleotide biosynthesis de novo is expected as the result of CTPS and IMPDH filamentation.

Finally, we demonstrate that the mutations in HsIMPDH1 associated to human retinopathies map into the allosteric nucleotide binding sites, which therefore alter the nucleotide-controlled conformational switch. Subsequently, they also disrupt allosteric inhibition mediated by GTP/GDP and modify the cytoophidia dynamics in vitro. Thereby, our data indicate that the HsIMPDH1 mutations within the first instance, will affect the intracellular levels of the purine nucleotide pools in retinal cells that, in a medium/long term, will lead to visual receptor cell death and subsequent retinal degeneration. Future studies are needed to elucidate the molecular details on how the alterations in the nucleotide pools develop into retinopathies. Moreover, it will also be very 
interesting to explore the potential physiological roles that cytoophidia play in IMPDH cytoophidia in the retina of adult mice as 1-2 $\mu \mathrm{m}$ long structures, mostly localized in the periphery of the retina, although they were detected throughout this organ (Supplemental Figure 8B). Altogether, these data indicate that cytoophidia form in the retina in vivo under normal developmental conditions and suggest a potential physiological role of cytoophidium that might be altered by the retinopathy-associated missense mutations of human IMPDH1.

In summary, our results provide novel and valuable information for understanding the physiological regulation of IMPDH as well as deciphering the putative roles of IMPDH cytoophidia in vivo, both under physiological and pathological conditions. Moreover, given that IMPDH is a widely validated drug target in several types of diseases, the reported results might eventually support future studies to determine the therapeutic potential of the cytoophidium as a useful target and/or biomarker.

\section{Materials and Methods}

Proteins and nucleotides. Expression and purification of IMPDH proteins was performed as previously described [7, 21]. Nucleotides were purchased from Sigma-Aldrich and Jena Bioscience. Missense mutations were introduced into the bacterial expression vectors by using QuickChange II Site-directed mutagenesis protocol (Agilent Technologies).

Protein polymerization analysis in vitro. Protein polymerization was monitored by turbidimetry at $340 \mathrm{~nm}$ on a spectrophotometer and/or light scattering at 410 $\mathrm{nm}$ on a spectrofluorimeter. Control experiments were also performed by pelleting the polymers by centrifugation and determining the protein concentration in pellets and supernatants. Samples of protein polymers were taken from each experiment for further analysis by electron microscopy (see below).

Enzyme kinetics assay. IMPDH activity was assayed, as previously reported [6], at $32^{\circ} \mathrm{C}$ using 96 or 384 -well microtiter plates by monitoring the reduction of $\mathrm{NAD}^{+}$and the subsequent increase in absorbance at $340 \mathrm{~nm}$. Human IMPDH 
enzymes at $100 \mu \mathrm{g} / \mathrm{mL}$ in buffer $100 \mathrm{mM}$ Tris- $\mathrm{HCl}, 100 \mathrm{mM} \mathrm{KCl}, 2 \mathrm{mM}$ DTT, $1 \mathrm{mM} \mathrm{MgCl} 2$ (final free $\mathrm{Mg}^{+2}$ concentration), $\mathrm{pH} 8.0$ were assayed using as substrates $0.5 \mathrm{mM} \mathrm{NAD}^{+}$and from 0.019 to $5 \mathrm{mM} \mathrm{IMP}$. The resulting experimental data were fitted by non-linear regression analysis to the MichaelisMenten and -allosteric sigmoidal- equations using GraphPad Prism (GraphPad Prism Software, Inc.).

To monitor enzyme kinetics under macromolecular crowding conditions, where turbidity might interfere with the measurement at $\lambda=340 \mathrm{~nm}$, the catalytic reaction was stopped by incubating the samples with $4 \mathrm{M}$ urea (final concentration) to disaggregate protein assemblies and avoid the turbidity of the solution. These experiments demonstrated that the constant background at $\lambda=340 \mathrm{~nm}$ due to the presence of turbidity in the solution could be easily subtracted from the raw measurements, without affecting the precise estimation of the kinetic parameters.

For control experiments, HsIMPDH1/2-WT and Y12A mutant enzymes under macromolecular crowding conditions were centrifuged at $5.000 \mathrm{~g}$ during 10 minutes at $25^{\circ} \mathrm{C}$, to pellet cytoophidia, and the catalytic activity of the supernatants was measured as described above.

Small Angle X-ray Scattering (SAXS). SAXS measurements were performed at the B21 beamline at the Diamond Light Source. Protein samples at $2.5 \mathrm{mg} / \mathrm{mL}$ in buffer $20 \mathrm{mM}$ TrisHCl, $300 \mathrm{mM} \mathrm{KCl}, 1 \mathrm{mM} \mathrm{MgCl}$ (final free $\mathrm{Mg}^{+2}$ concentration), $3 \mathrm{mM}$ DTT, $\mathrm{pH}$ 8.0, were measured in the presence of increasing amounts of nucleotides (total concentration of nucleotides ranging from 0.02 to $3 \mathrm{mM}$ ). Experimental data were reduced following standard methodologies by using the program PRIMUS. The fractions of tetramers, expanded and compacted octamers were calculated with the program OLIGOMER and the theoretical solution scattering profiles with CRYSOL. All these programs are found within the ATSAS software suite [29].

Crystallization and structure determination. Crystals of the complexes HsIMPDH2-GTP and HsIMPDH2-GDP were grown at $22^{\circ} \mathrm{C}$ using the vapor diffusion method by mixing a protein solution at $7.5 \mathrm{mg} / \mathrm{ml}$ in $10 \mathrm{mM}$ Tris-HCl, $150 \mathrm{mM} \mathrm{KCl}, 1 \mathrm{mM}$ DTT, $7.5 \mathrm{mM}$ GTP (or GDP), $5 \mathrm{mM}$ total $\mathrm{MgCl}_{2}, \mathrm{pH}$ 8.0, 
with an equal volume of mother liquor consisting of $25 \%$ PEG-1500, $0.1 \mathrm{M}$ buffer MIB (malonic acid, imidazole and boric acid), pH 9.0, for HsIMPDH-GTP and $0.1 \mathrm{M}$ sodium citrate, $\mathrm{pH}$ 5.5, $0.2 \mathrm{M}$ lithium sulfate and $15 \%$ ethanol, for HsIMPDH2-GDP. Protein crystals were flashed-cooled in liquid nitrogen and data were collected at $100 \mathrm{~K}$, using monochromatic X-rays of $\approx 1 \AA$ A wavelength, at the MX beamlines of the Diamond Light Source synchrotron (UK) or the XALOC beamline in the ALBA synchrotron (Spain). Crystals of HsIMPDH2GDP were cryo-protected by immersion in $\mathrm{NVH}$ oil before being flashed-cooled in liquid nitrogen. Diffraction intensities were indexed and integrated by using the software autoPROC [30].

The experimental data were phased by molecular replacement using the program PHASER [31] using as template the structure of the complex AgIMPDH-GDP (PDB code 4Z87). The structures were iteratively refined by alternating manual modelling with COOT [32] with the automated refinement utility of the PHENIX crystallographic software suite [33]. Rigid body, gradientdriven positional, restrained individual isotropic B-factor and TLS were used for structure refinement.

Electron microscopy. Samples were analyzed by electron microscopy after being adsorbed to glow-discharged carbon coated grids and stained with 2\% uranyl acetate. Grids were observed using a JEOL JEM-1230 transmission electron microscope operated at $100 \mathrm{kV}$ and a nominal magnification of 40.000 . Images were taken under low dose conditions with a CMOS Tvips TemCamF416 camera, at $2.84 \AA$ per pixel. Image processing was performed using SCIPION package [34]. The contrast transfer function of the microscope for each micrograph was estimated using CTFFIND4 [35]. Helical segments were picked from the micrographs using the Xmipp3 manual-picking routine implemented inside SCIPION.

In the initial step, images of the helical segments were extracted and horizontally pre-aligned using a multi-reference pattern-free alignment protocol [36]. After pre-alignment images were two-dimensional classified using RELION software [37] limiting in-plane rotations, allowing bimodal search and using a rectangular mask to focus the classification process in the helix. Classes containing the straightest particles were selected for 3D reconstruction. Initial 
model was built by assigning random rotation angles to the images and $3 D$

\section{Accession numbers}


The atomic coordinates and the structure factors described in this work have been deposited in the Research Collaboratory for Structural Bioinformatics Protein Data Bank under the codes PDB ID: 6100 (HsIMPDH2-GTP) and PDB ID: 6IOM (HsIMPDH2-GDP). The electron microscopy-derived maps have been deposited in the Electron Microscopy Data Bank under the accession codes EMD ID: 4402 (HsIMPDH1-GDP) y EMD ID: 4403 (HsIMPDH1-ATP). 


\section{Acknowledgements}

This work was supported by the Spanish Ministerio de Ciencia, Innovación y Universidades (grants BFU2016-79237-P to R.M.B and BIO2014-56930-P to J.L.R.). D.F.J. was supported by a pre-doctoral contract from the "Junta de Castilla y León". We thank María Dolores Sánchez, Silvia Domínguez and Marta Santos for excellent technical help, Ángel Hernández for help with cell cultures and José $M$ de Pereda and Kyle B del Valle for their valuable comments and suggestions to the manuscript. Protein crystallography experiments were performed at the i03 beamline (Diamond Light Source, UK) and XALOC (ALBA Synchrotron, Spain) beamlines with the collaboration of Diamond and ALBA staff. X-ray scattering experiments were performed at the B21- Solution State SAXS beamline (Diamond Light Source, UK). 


\section{Figure captions}

Figure 1. The conformational switch of HsIMPDH controls its catalytic activity. A-B. Experimental SAXS profiles of HsIMPDH1 (A) and HsIMPDH2 (B) wildtype and mutant $Y 12 A$ in the absence and presence of the indicated purine nucleotides. C-D. Plots representing the fraction of compact octamers, as determined by SAXS, (empty symbols and dashed lines) and the catalytic activity $\left(\mathrm{V}_{\max }\right.$ app / $\mathrm{V}_{\text {max }}$; filled symbols and continuous lines) versus GDP concentration for HsIMPDH1-Y12A (C) and HsIMPDH2-Y12A (D). Squares: 3 mM ATP fixed concentration versus increasing concentrations of GDP. Circles: increasing concentrations of GDP in the absence of ATP. Data points represent the mean value and the standard errors. Lines represent the non-linear regression analysis of the experimental data, according to a standard allosteric sigmoidal equation (GraphPad Prism). At the fixed concentrations of ATP ( 3 $\mathrm{mM})$ and GDP ( $3 \mathrm{mM})$ used for these experiments, HsIMPDH1-Y12A and HsIMPDH2-Y12A proteins remained as octamers with no detectable fraction of tetramers, as determined by SAXS.

Figure 2. Retinopathy-associated missense mutations disrupt GTP/GDPmediated allosteric inhibition in vitro. Normalized catalytic activity $\left(\mathrm{V}_{\max }\right.$ derived from the Michaelis-Menten analysis of the experimental data) of HsIMPDH1-WT and retinopathy-associated mutants in the absence or presence of purine nucleotides. Error bars represent standard errors.

Figure 3. In vitro reconstitution of human IMPDH cytoophidia assembly. A. Turbidity measurements, indicating the formation of large polymers of HsIMPDH2, at different Ficoll-70 concentrations in the presence or absence of purine nucleotides. B. Electron microscopy analysis of the samples in the absence (left panels) and presence (right panels) of $150 \mathrm{~g} / \mathrm{L}$ Ficoll-70. Upper panels: no nucleotide; middle panels: $3 \mathrm{mM}$ ATP; lower panels: $5 \mathrm{mM}$ GDP. Scale bars are 100 nanometer long. The red rectangle in the upper right panel shows bundles of twisted and curved filaments that intricate into the three dimensions without apparent regular or defined structure, while the blue rectangle shows ordered bundles of parallel protofilaments that were sporadically observed. C. Turbidity measurements of purified IMPDH from different organisms. HsIMPDH1, Homo sapiens isoform 1; HsIMPDH2: Homo 
sapiens isoform 2; AgIMPDH: Ashbya gossypii; EcIMPDH: Escherichia coli; BsIMPDH: Bacillus subtilis. Error bars represent standard errors.

Figure 4. Retinopathy-associated missense mutations alter IMPDH cytoophidia assembly in vitro. Turbidity measurements, indicating the formation of large polymers in vitro, at $150 \mathrm{~g} / \mathrm{L}$ Ficoll-70 of HsIMPDH1 wild-type and retinopathyassociated mutants in the absence or presence of purine nucleotides. Data were taken 10 minutes after dilution of the proteins into a solution containing Ficoll-70 and the indicated nucleotides. Error bars represent standard errors.

Figure 5. Human IMPDH cytoophidia are catalytically active and resistant to GDP allosteric inhibition. A-B. Michaelis-Menten representation of the catalytic activity of HsIMPDH1 (A) and HsIMPDH2 (B) wild-type (squares) and Y12A mutant (circles) in a dilute solution (solid Figures and continuous green lines) and in the presence of $150 \mathrm{~g} / \mathrm{L}$ Ficoll-70 (empty Figures and discontinuous red lines). C-D. Plots representing normalized solution turbidity (dotted lines) and catalytic activity $\left(\mathrm{V}_{\max }{ }^{\text {app }} / \mathrm{V}_{\max }\right.$; solid Figures and continuous green line in the absence of Ficoll-70, empty Figures and dashed red line for the short incubation with Ficoll-70 and empty Figures and dashed blue line for the long incubation with Ficoll-70) at different GDP concentrations for HsIMPDH1-WT (C) and HsIMPDH2-WT (D). In our experimental setup, the measurements were performed approximately 30 minutes after GDP addition. Lines represent the non-linear regression analysis of the experimental data, according to a standard allosteric sigmoidal equation (GraphPad Prism). Error bars represent standard errors.

Figure 6. The nucleotide controlled-conformational switch of human IMPDH. Upper panels: the three different octamer conformations observed in eukaryotic IMPDH enzymes and their respective PDB codes. IMPDH protein is shown in cartoons and nucleotides in sticks. The approximate longitudinal dimensions of the octamers are indicated on their side. Middle panels: close-up view of two interacting Bateman domains from adjacent tetramers within the octameric arrangement. The black dashed lines show the approximate longitudinal axis of helices comprising residues 194-204. Lower panels: schematic representation of the assembly of human IMPDH into different polymers. 


\section{References}

[1] Hedstrom L. IMP dehydrogenase: structure, mechanism, and inhibition. Chemical reviews. 2009;109:2903-28.

[2] Labesse G, Alexandre T, Vaupré L, Salard-Arnaud I, Him J, Raynal B, et al. MgATP Regulates Allostery and Fiber Formation in IMPDHs. Structure (London, England : 1993). 2013;21:975-85.

[3] Labesse G, Alexandre T, Gelin M, Haouz A, Munier-Lehmann H. Crystallographic studies of two variants of Pseudomonas aeruginosa IMPDH with impaired allosteric regulation. Acta crystallographica Section D, Biological crystallography. 2015;71:1890-9.

[4] Ji Y, Gu J, Makhov A, Griffith J, Mitchell B. Regulation of the interaction of inosine monophosphate dehydrogenase with mycophenolic Acid by GTP. The Journal of biological chemistry. 2006;281:206-12.

[5] Anthony SA, Burrell AL, Johnson MC, Duong-Ly KC, Kuo YM, Simonet JC, et al. Reconstituted IMPDH polymers accommodate both catalytically active and inactive conformations. Molecular biology of the cell. 2017.

[6] Buey RM, Fernandez-Justel D, Marcos-Alcalde I, Winter G, Gomez-Puertas $\mathrm{P}$, de Pereda JM, et al. A nucleotide-controlled conformational switch modulates the activity of eukaryotic IMP dehydrogenases. Sci Rep. 2017;7:2648.

[7] Buey RM, Ledesma-Amaro R, Velazquez-Campoy A, Balsera M, Chagoyen $\mathrm{M}$, de Pereda JM, et al. Guanine nucleotide binding to the Bateman domain mediates the allosteric inhibition of eukaryotic IMP dehydrogenases. Nat Commun. 2015;6:8923.

[8] McGrew DA, Hedstrom L. Towards a pathological mechanism for IMPDH1linked retinitis pigmentosa. Advances in experimental medicine and biology. 2012;723:539-45.

[9] Pedley AM, Benkovic SJ. A New View into the Regulation of Purine Metabolism: The Purinosome. Trends in biochemical sciences. 2017;42:141-54. [10] Carcamo WC, Satoh M, Kasahara H, Terada N, Hamazaki T, Chan JY, et al. Induction of cytoplasmic rods and rings structures by inhibition of the CTP and GTP synthetic pathway in mammalian cells. PLoS One. 2011;6:e29690.

[11] Chang CC, Lin WC, Pai LM, Lee HS, Wu SC, Ding ST, et al. Cytoophidium assembly reflects upregulation of IMPDH activity. J Cell Sci. 2015;128:3550-5.

[12] Aughey GN, Liu JL. Metabolic regulation via enzyme filamentation. Crit Rev Biochem Mol Biol. 2015;51:282-93.

[13] Wang H, Saho Q-X. The cytoophidium: A novel intracellular compartmentation formed by metabolic enzymes. Academia Journal of Biotechnology. 2017;5:7.

[14] Keppeke GD, Chang CC, Peng M, Chen LY, Lin WC, Pai LM, et al. IMP/GTP balance modulates cytoophidium assembly and IMPDH activity. Cell Div. 2018;13:5.

[15] Duong-Ly KC, Kuo YM, Johnson MC, Cote JM, Kollman JM, Soboloff J, et al. $T$ cell activation triggers reversible inosine-5'-monophosphate dehydrogenase assembly. J Cell Sci. 2018;131.

[16] Calise SJ, Carcamo WC, Krueger C, Yin JD, Purich DL, Chan EK. Glutamine deprivation initiates reversible assembly of mammalian rods and rings. Cell Mol Life Sci. 2014;71:2963-73. 
[17] Calise SJ, Purich DL, Nguyen T, Saleem DA, Krueger C, Yin JD, et al. 'Rod and ring' formation from IMP dehydrogenase is regulated through the onecarbon metabolic pathway. J Cell Sci. 2016;129:3042-52.

[18] Chang CC, Keppeke GD, Sung LY, Liu JL. Interfilament interaction between IMPDH and CTPS cytoophidia. FEBS J. 2018;285:3753-68.

[19] Juda P, Smigova J, Kovacik L, Bartova E, Raska I. Ultrastructure of cytoplasmic and nuclear inosine-5'-monophosphate dehydrogenase 2 "rods and rings" inclusions. J Histochem Cytochem. 2014;62:739-50.

[20] Liu JL. The Cytoophidium and Its Kind: Filamentation and Compartmentation of Metabolic Enzymes. Annu Rev Cell Dev Biol. 2016;32:349-72.

[21] Thomas E, Gunter J, Webster J, Schieber N, Oorschot V, Parton R, et al. Different characteristics and nucleotide binding properties of inosine monophosphate dehydrogenase (IMPDH) isoforms. PloS one. 2012;7:12.

[22] Nimmesgern E, Fox T, Fleming MA, Thomson JA. Conformational changes and stabilization of inosine 5'-monophosphate dehydrogenase associated with ligand binding and inhibition by mycophenolic acid. The Journal of biological chemistry. 1996;271:19421-7.

[23] Gunter JH, Thomas EC, Lengefeld N, Kruger SJ, Worton L, Gardiner EM, et al. Characterisation of inosine monophosphate dehydrogenase expression during retinal development: differences between variants and isoforms. Int $\mathrm{J}$ Biochem Cell Biol. 2008;40:1716-28.

[24] Shen QJ, Kassim H, Huang Y, Li H, Zhang J, Li G, et al. Filamentation of Metabolic Enzymes in Saccharomyces cerevisiae. J Genet Genomics. 2016;43:393-404.

[25] Vopel T, Makhatadze Gl. Enzyme activity in the crowded milieu. PLoS One. 2012;7:e39418.

[26] Lynch EM, Hicks DR, Shepherd M, Endrizzi JA, Maker A, Hansen JM, et al. Human CTP synthase filament structure reveals the active enzyme conformation. Nat Struct Mol Biol. 2017;24:507-14.

[27] Rivas G, Minton AP. Macromolecular Crowding In Vitro, In Vivo, and In Between. Trends in biochemical sciences. 2016;41:970-81.

[28] Minton AP. The influence of macromolecular crowding and macromolecular confinement on biochemical reactions in physiological media. The Journal of biological chemistry. 2001;276:10577-80.

[29] Franke D, Petoukhov MV, Konarev PV, Panjkovich A, Tuukkanen A, Mertens HDT, et al. ATSAS 2.8: a comprehensive data analysis suite for smallangle scattering from macromolecular solutions. Journal of applied crystallography. 2017;50:1212-25.

[30] Vonrhein C, Flensburg C, Keller P, Sharff A, Smart O, Paciorek W, et al. Data processing and analysis with the autoPROC toolbox. Acta crystallographica Section D, Biological crystallography. 2011;67:293-302.

[31] McCoy AJ, Grosse-Kunstleve RW, Adams PD, Winn MD, Storoni LC, Read RJ. Phaser crystallographic software. Journal of applied crystallography. 2007;40:658-74.

[32] Emsley P, Lohkamp B, Scott WG, Cowtan K. Features and development of Coot. Acta crystallographica Section D, Biological crystallography. 2010;66:486501.

[33] Adams PD, Afonine PV, Bunkoczi G, Chen VB, Davis IW, Echols N, et al. PHENIX: a comprehensive Python-based system for macromolecular structure 
solution. Acta crystallographica Section D, Biological crystallography. 2010;66:213-21.

[34] de la Rosa-Trevin JM, Quintana A, Del Cano L, Zaldivar A, Foche I, Gutierrez J, et al. Scipion: A software framework toward integration, reproducibility and validation in 3D electron microscopy. J Struct Biol. 2016;195:93-9.

[35] Rohou A, Grigorieff N. CTFFIND4: Fast and accurate defocus estimation from electron micrographs. J Struct Biol. 2015;192:216-21.

[36] Sorzano CO, Bilbao-Castro JR, Shkolnisky Y, Alcorlo M, Melero R, Caffarena-Fernandez G, et al. A clustering approach to multireference alignment of single-particle projections in electron microscopy. J Struct Biol. 2010;171:197-206.

[37] Scheres SH. RELION: implementation of a Bayesian approach to cryo-EM structure determination. J Struct Biol. 2012;180:519-30. 
A

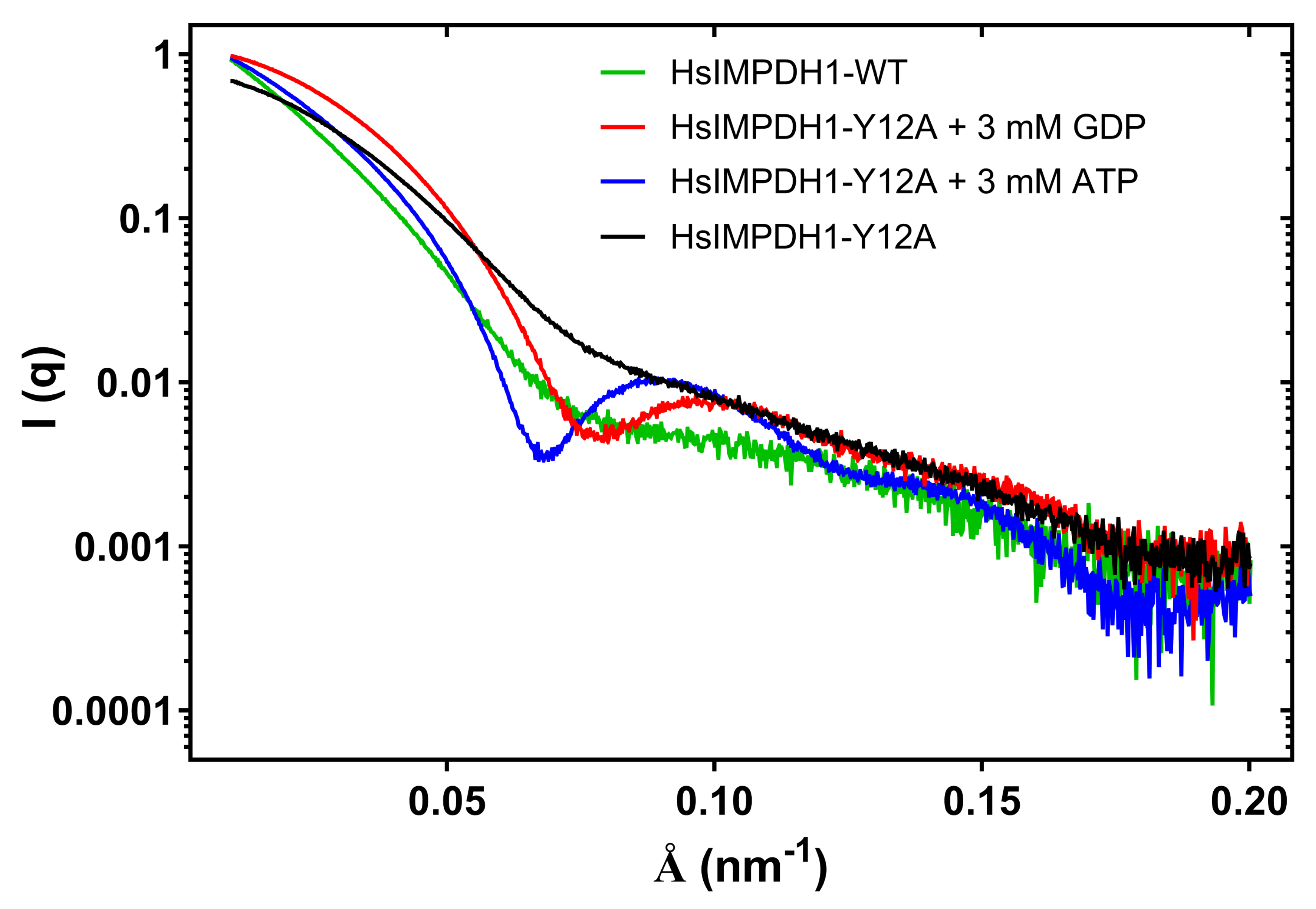

C

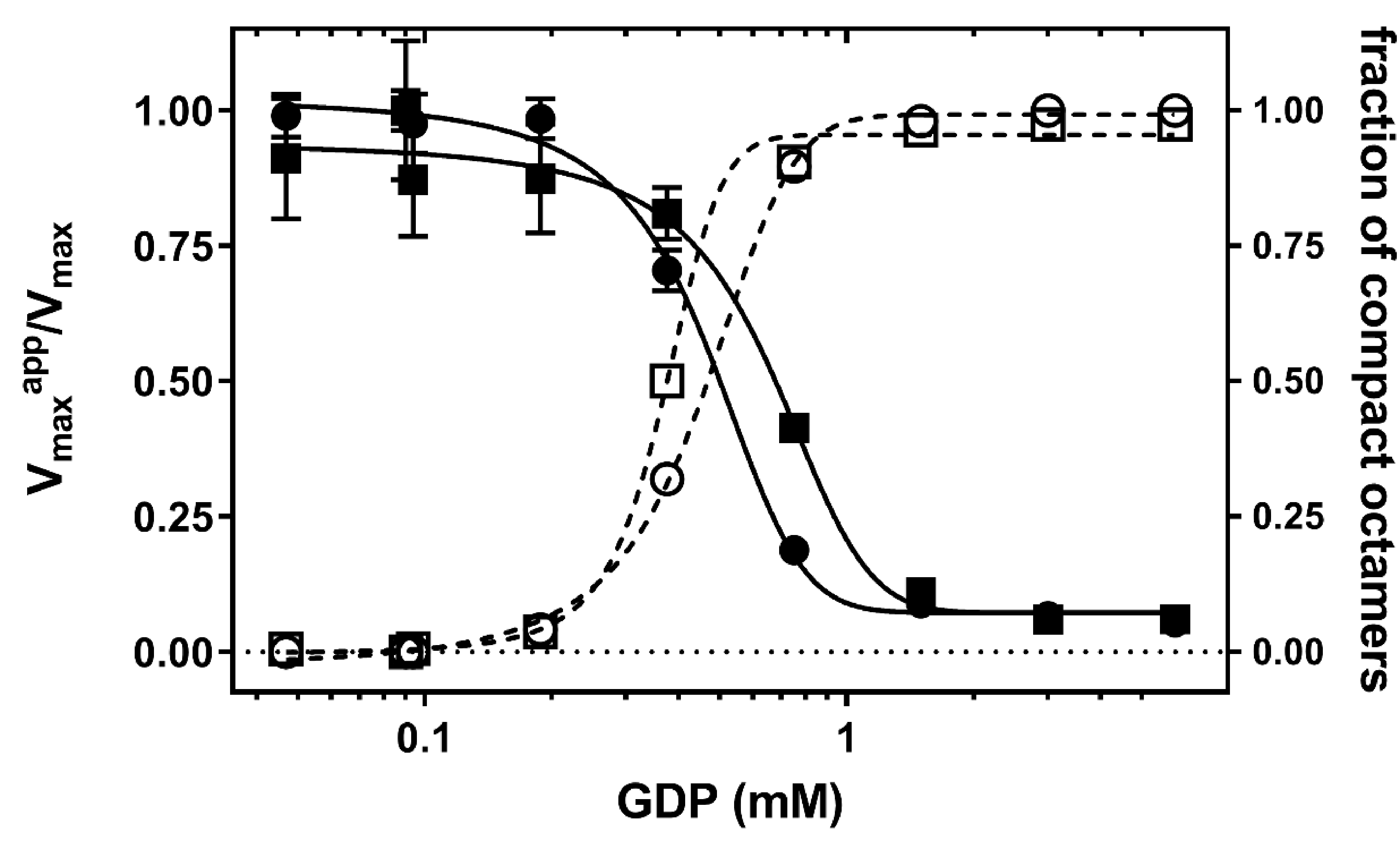

B

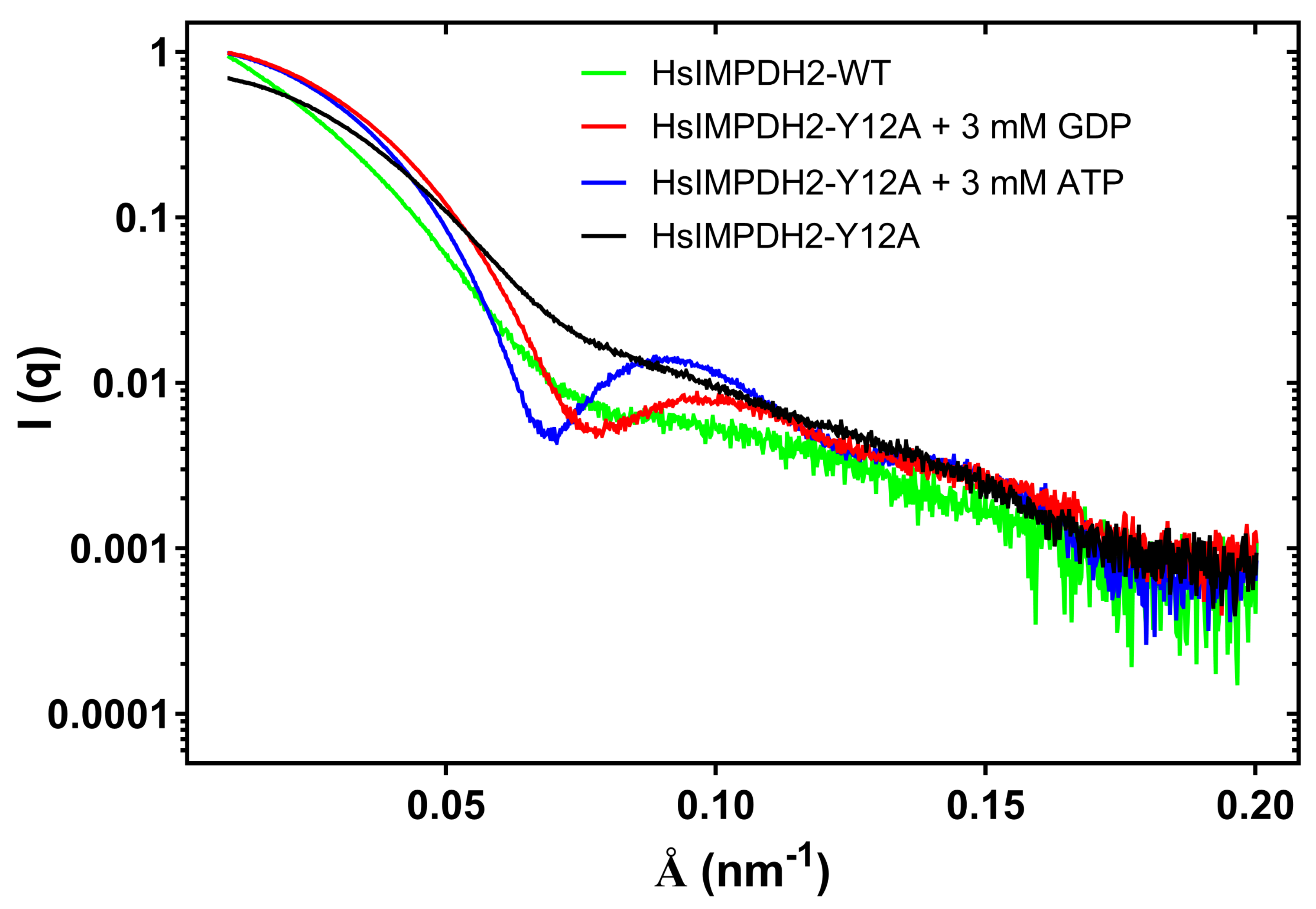

D

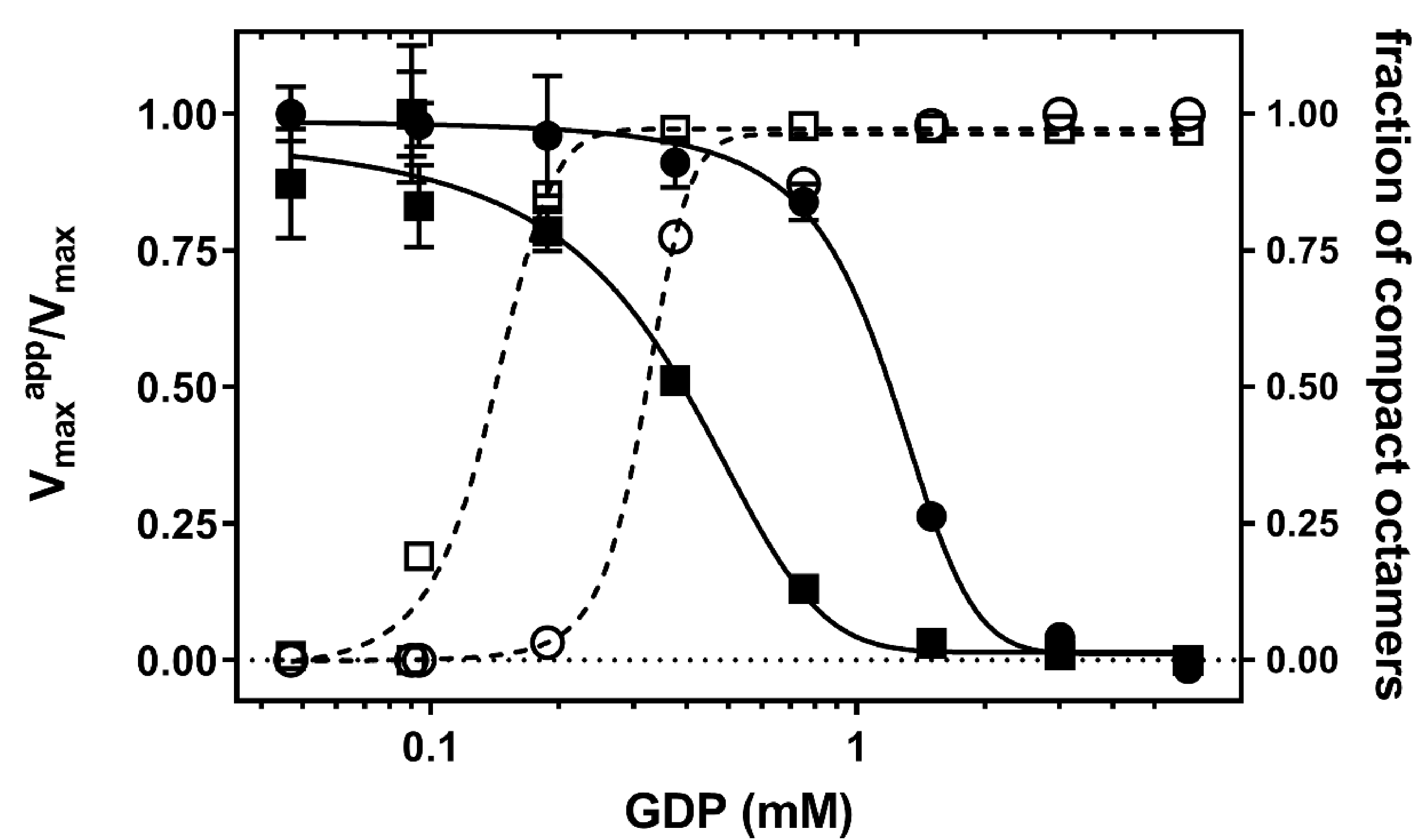


FIGURE 2

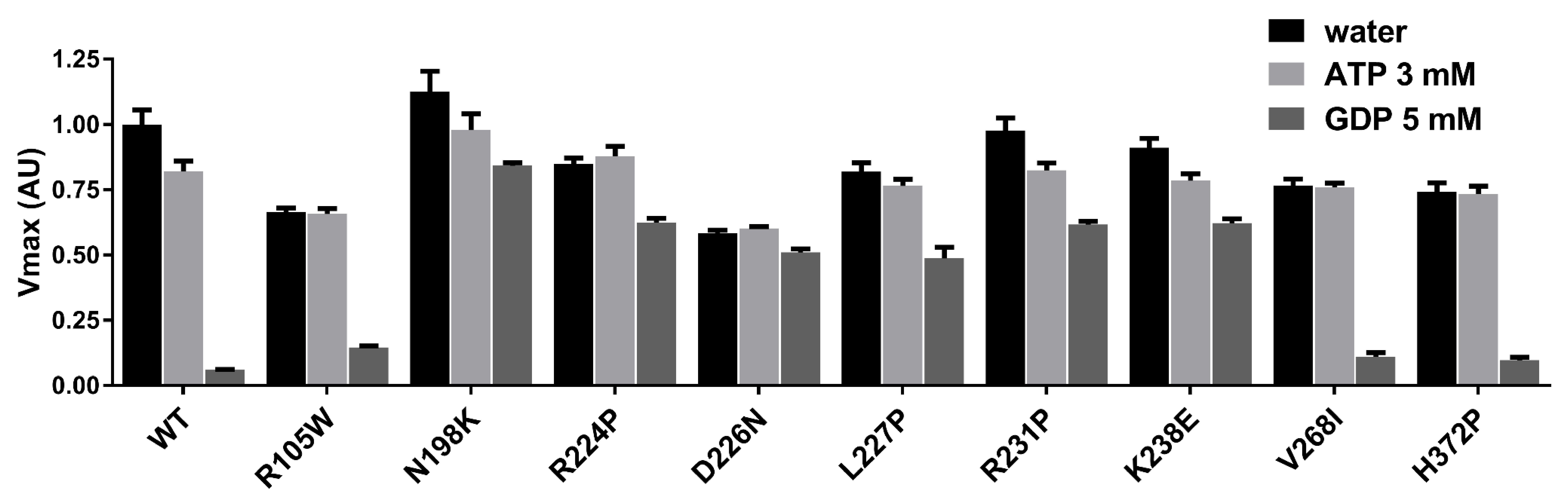


FIGURE 3

A

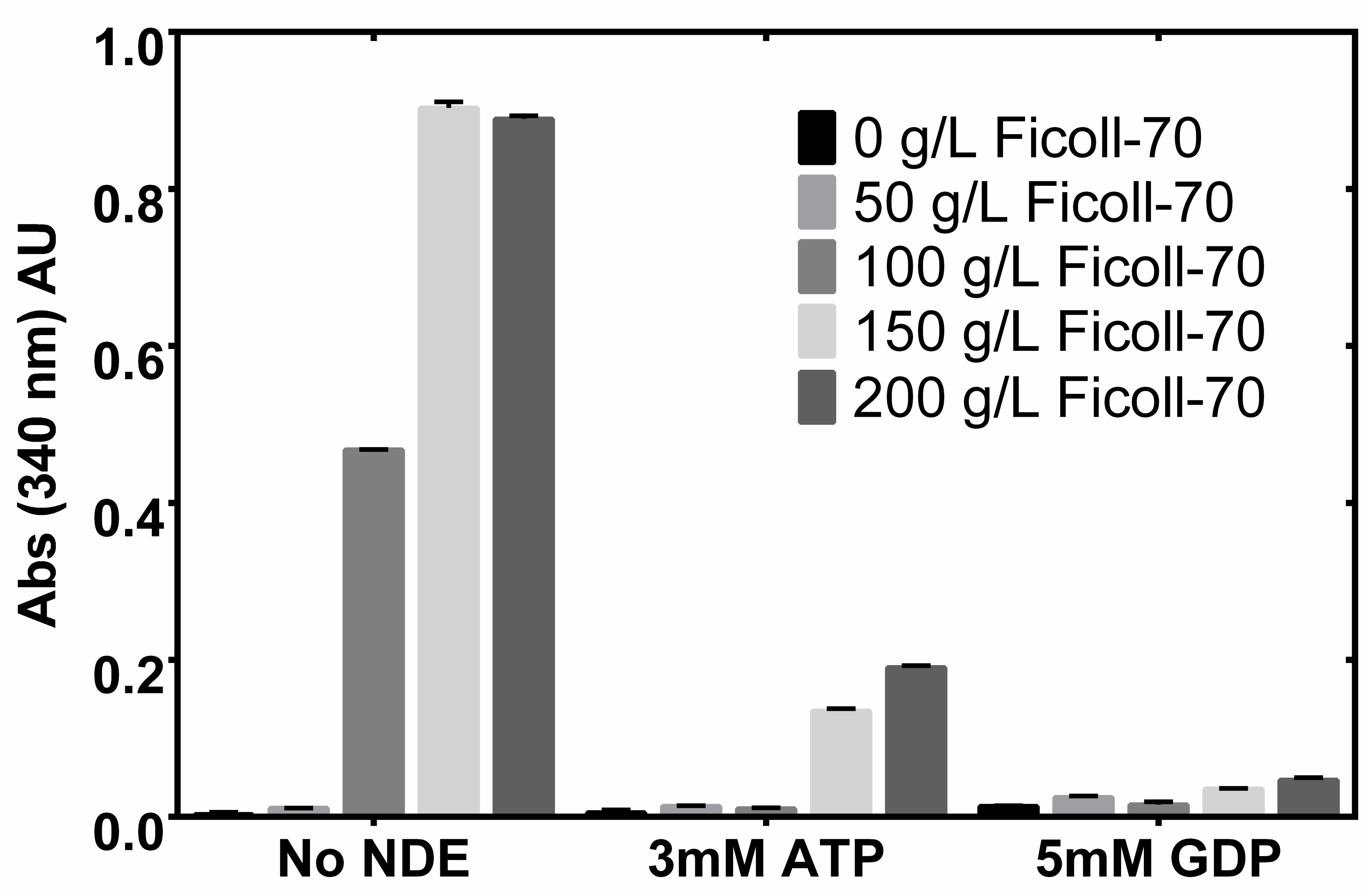

B
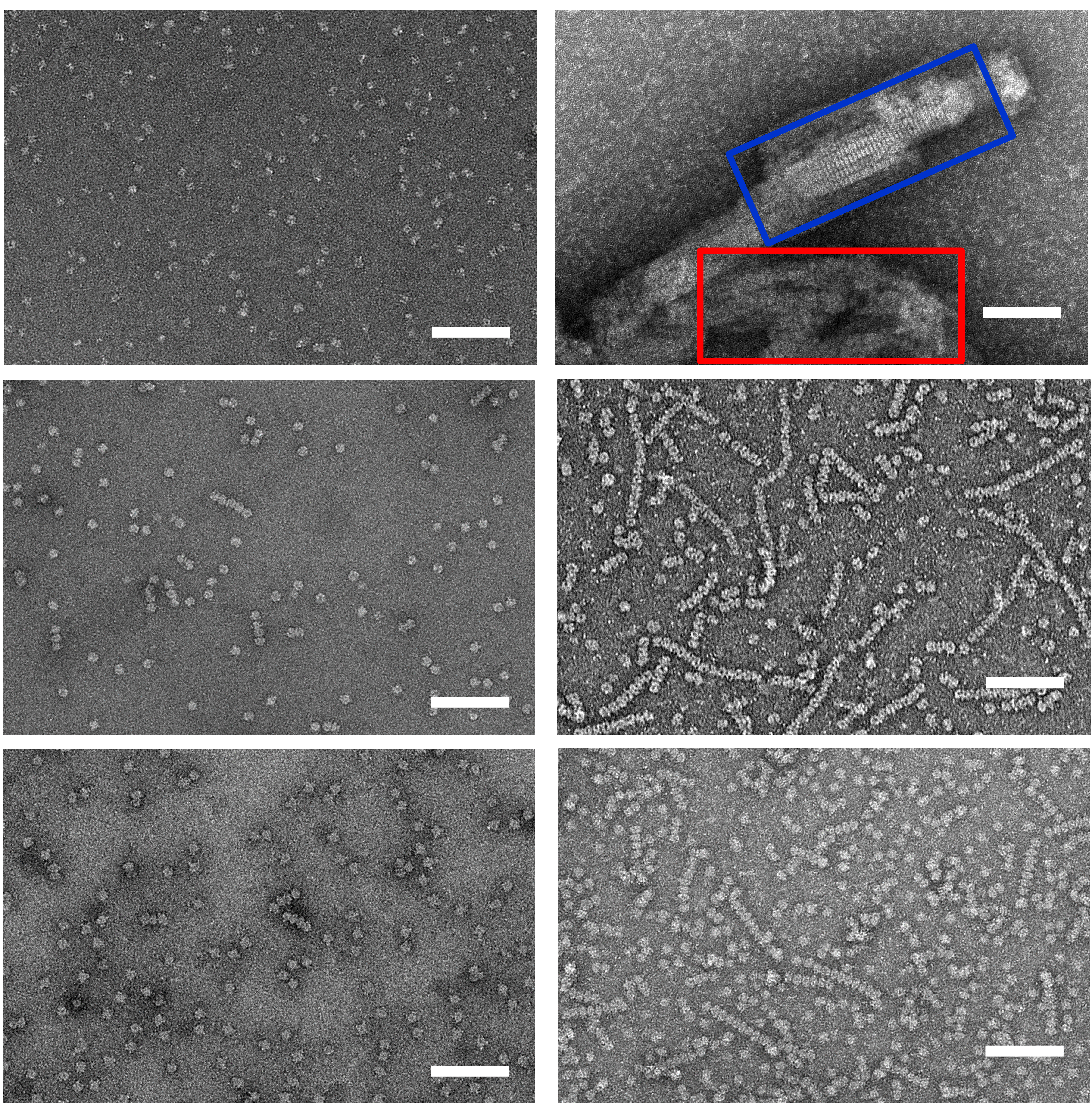

C

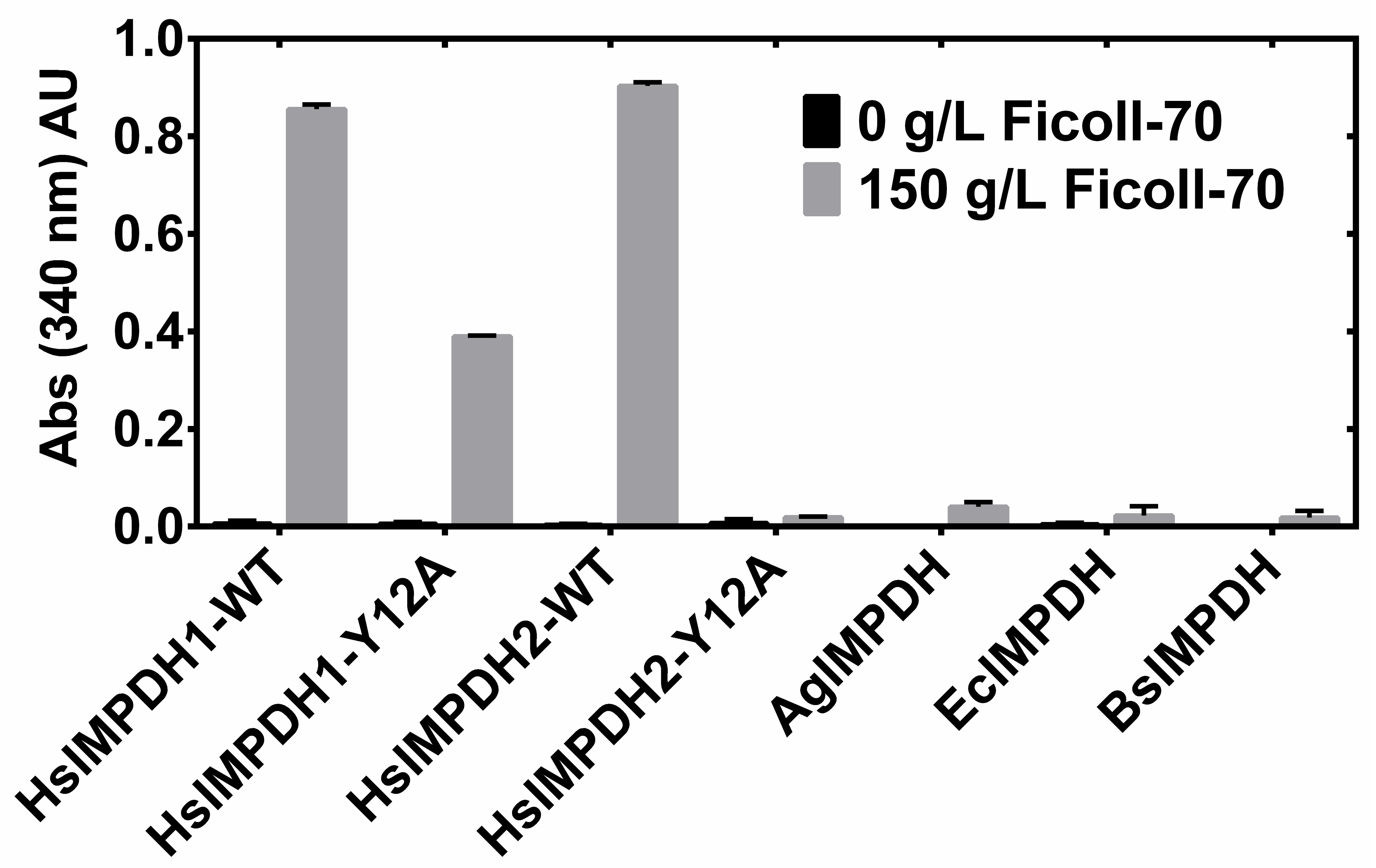


FIGURE 4

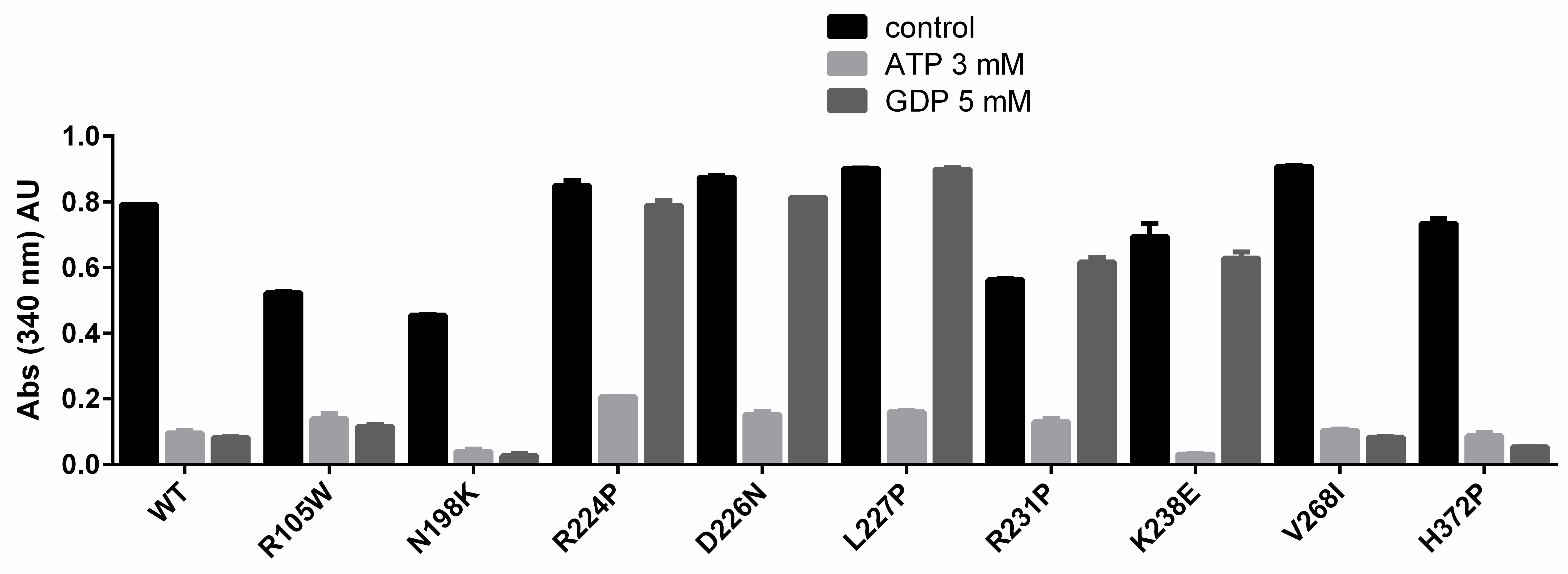




\section{A}

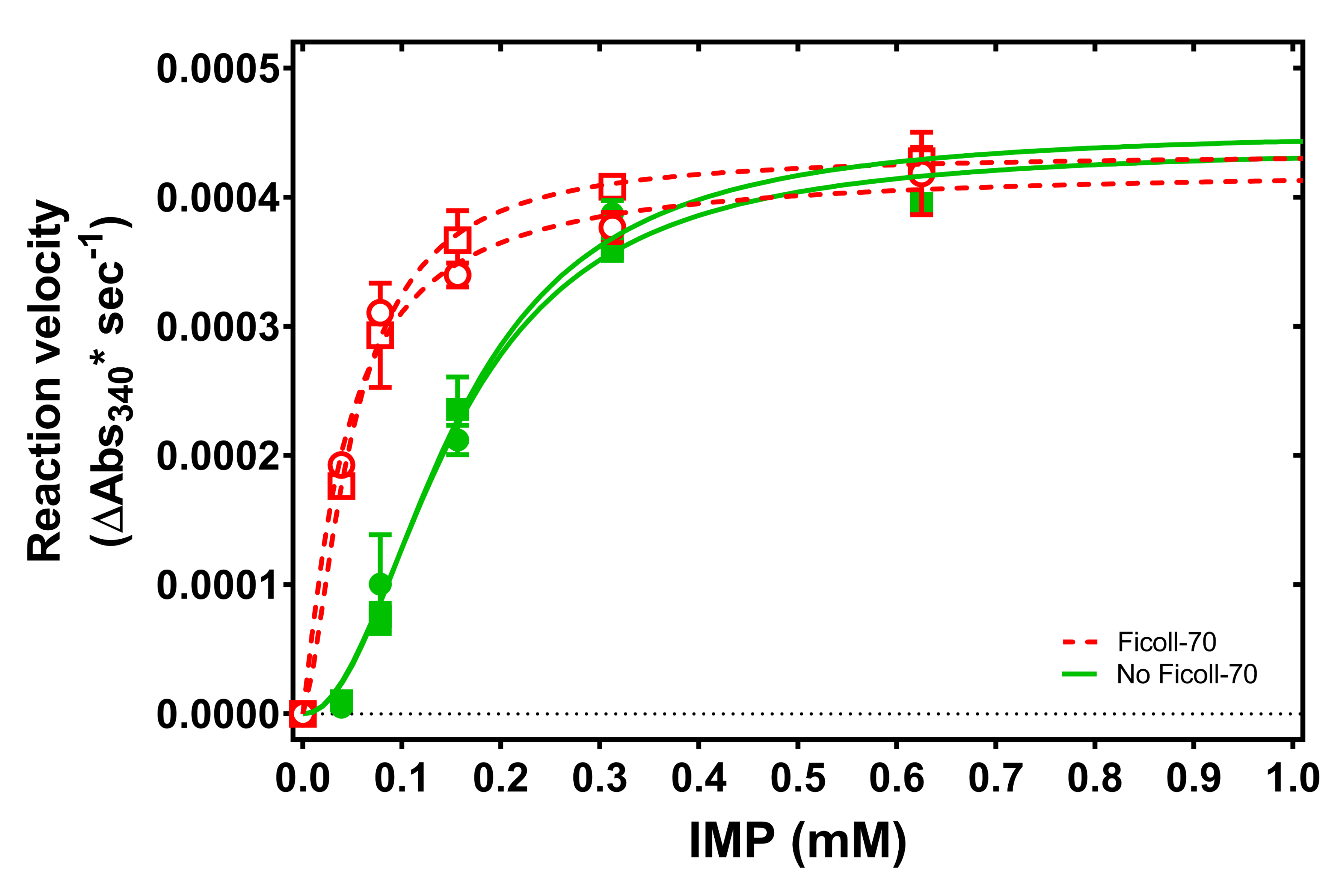

\section{C}

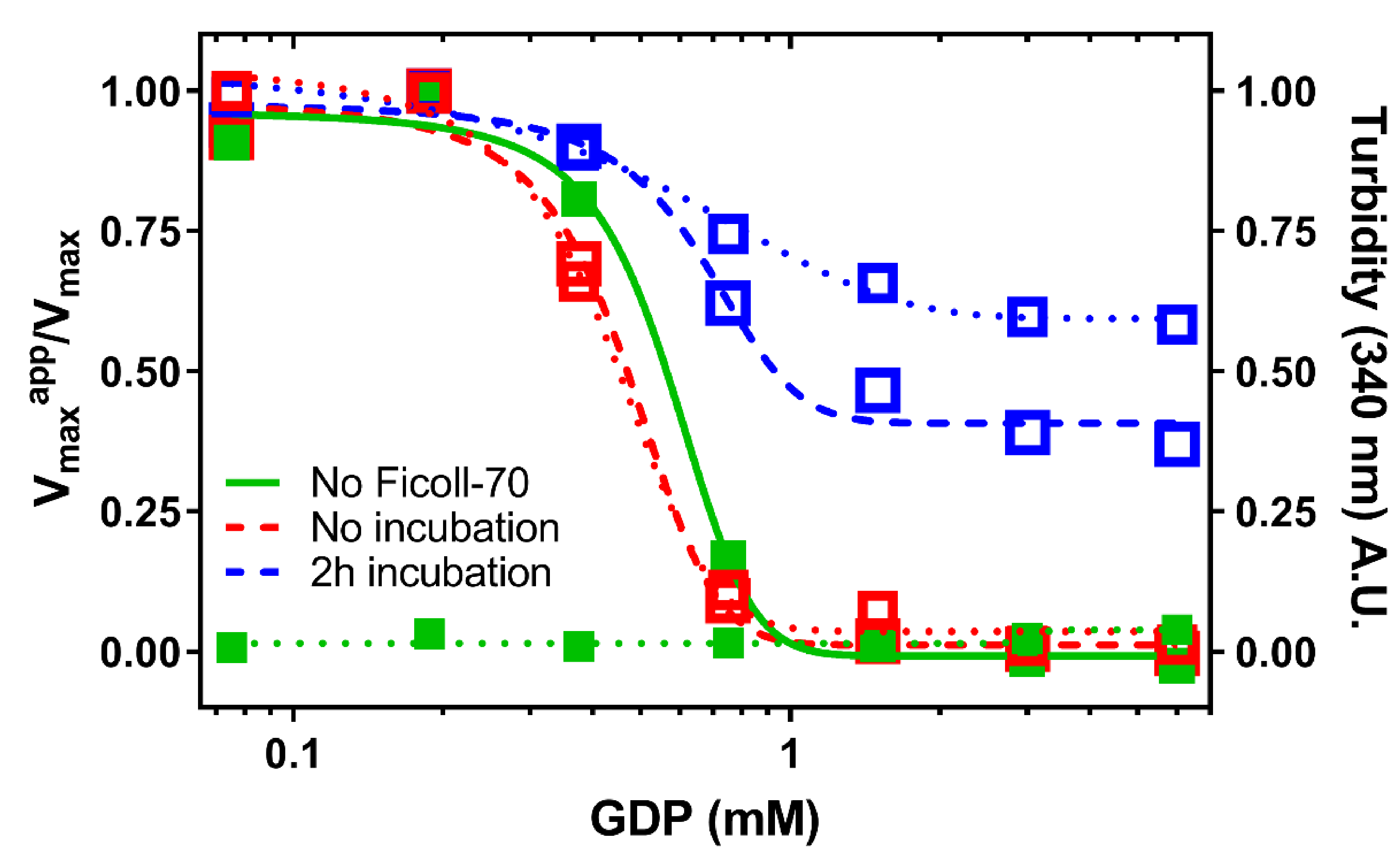

B

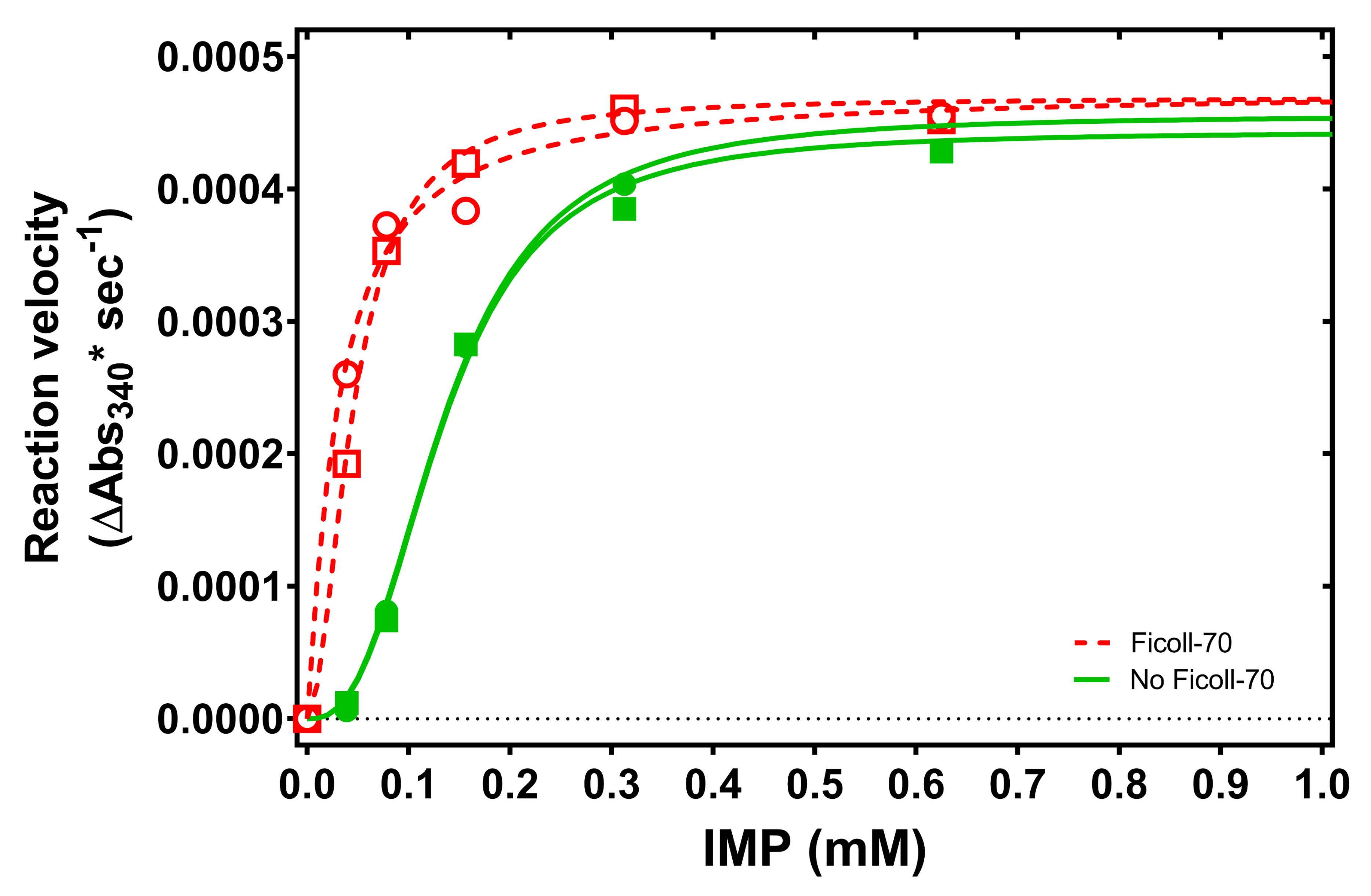

D

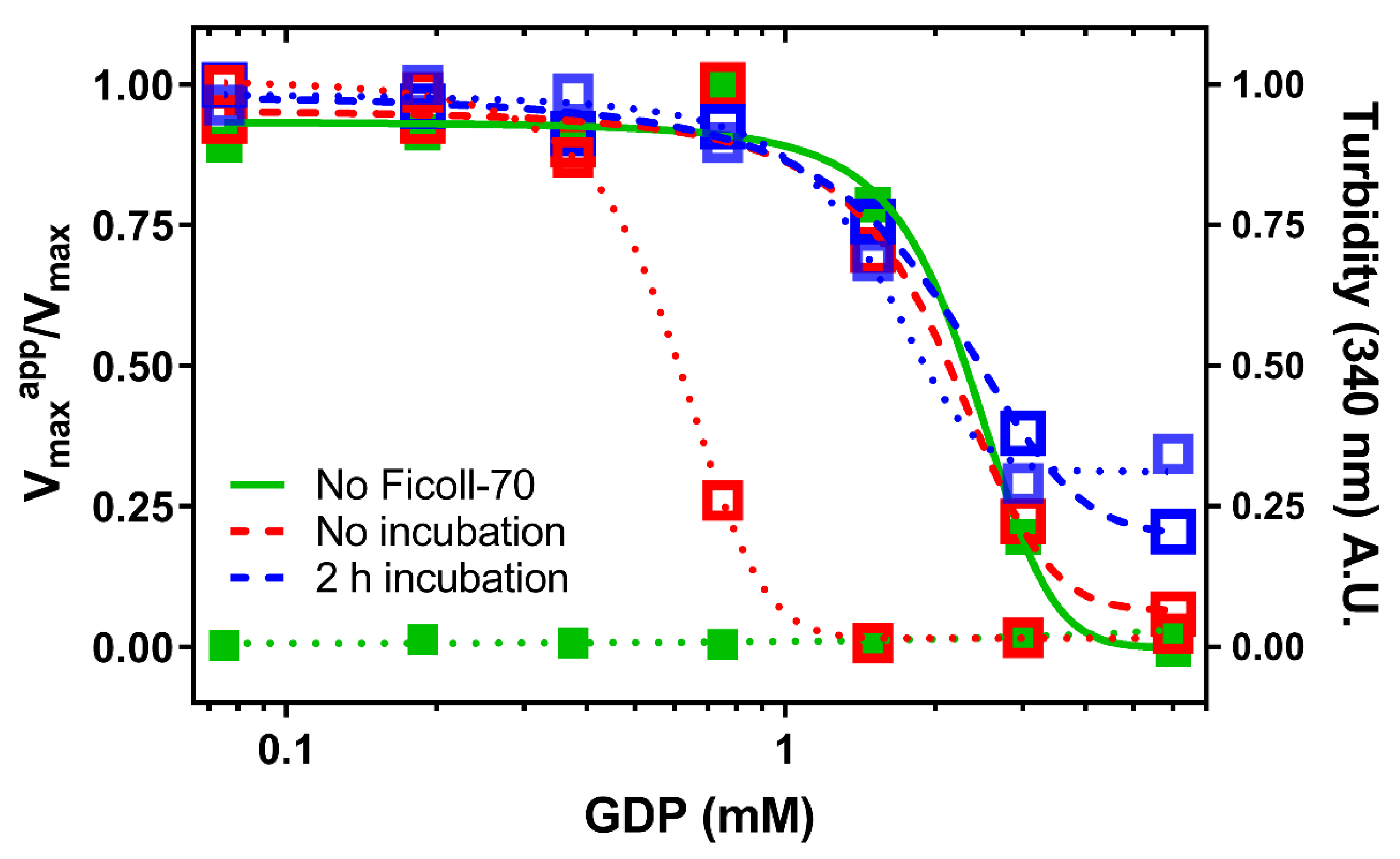




\section{FIGURE 6}
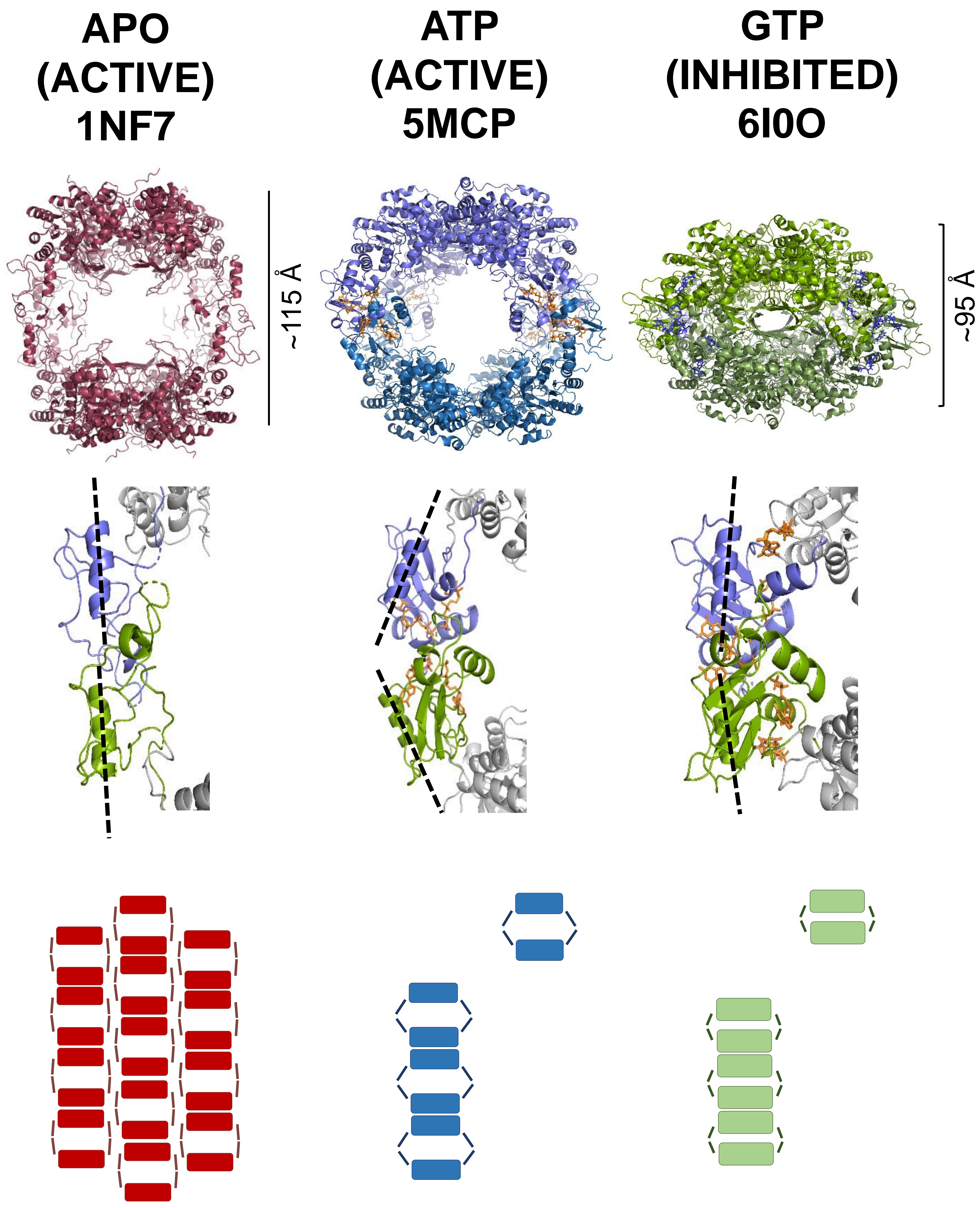

Supplementary Material (To be Published)
Click here to download Supplementary Ma

Click here to download Supplementary Material (To be Published): SupplementalData.pdf 